10.5005/jp-journals-10070-0014

\title{
A Rare Case of Inguinal Canal Malignancy
}

\author{
${ }^{1}$ Rahul Goel, ${ }^{2}$ Kasiviswanathan M
}

\begin{abstract}
Background: The commonest differentials in a case of inguinal swelling are a hernia, hydrocele, lipoma and lymph nodal mass. However, we may come across some rare causes of inguinal lumps such as leiomyosarcoma. This is a case of an elderly gentleman who presented with a history of a painless, progressively enlarging mass over the right inguinal region which on further investigation was found to be a malignant tumour.

Case presentation: A 70-year-old male patient presented with a mass over the right inguinal region $7 \times 7 \times 8 \mathrm{~cm}$ in size. It was nonreducible and non-trans illuminant. There was no cough impulse either. Sonography of the lump revealed a heterogeneous mass originating in the right inguinal canal queried to be an enlarged lymph node. Computed tomography (CT) scan of the abdomen and pelvis had similar findings. Fine needle aspiration cytology (FNAC) of the lump was inconclusive. The patient underwent an excision biopsy, and intraoperatively a huge fleshy tumour originating from the spermatic cord structures was visualized. Histopathological examination was suggestive of a grade II leiomyosarcoma.
\end{abstract}

Conclusion: Spermatic cord leiomyosarcoma, although rare, should be kept in mind when dealing with an unusual lump in the cord. This article reviews the literature regarding paratesticular leiomyosarcoma presentation, diagnosis, and treatment.

Keywords: Inguinal canal, Leiomyosarcoma, Malignancy.

How to cite this article: Goel R, Kasiviswanathan M. A Rare Case of Inguinal Canal Malignancy. Journal of Medical Academics 2018;1(1):68-71.

Source of support: Nil

Conflict of interest: None

\section{INTRODUCTION}

The term "sarcoma" is derived from the Greek word sarx meaning "fleshy." It is used to include the malignant mesenchymal tumours of the body. This is in contrast to malignancies arising from epithelial cells, which are called "carcinoma."

Sarcomas can be grouped into two main kinds: soft tissue sarcomas and bone sarcomas (e.g., osteosarcomas). The nomenclature of sarcomas is as per the tissue they resemble, for example, Fibrosarcomas bear a resemblance

\footnotetext{
${ }^{1}$ Senior Resident, ${ }^{2}$ Resident

${ }^{1}$ Department of General Surgery, Armed Forces Medical College, Pune, Maharashtra, India

${ }^{2}$ Department of Radiodiagnosis and Imaging, Base Hospital Delhi Cantonment, Delhi, India

Corresponding Author: Rahul Goel, Senior Resident, Department of General Surgery, Armed Forces Medical College, Pune, Maharashtra, India, Phone: +917263811480 , e-mail: unknownsince1986@yahoo.co.in
}

to fibrous tissue; leiomyosarcomas-smooth muscle cells, and so on. Earlier, it was postulated that sarcomas arise from the malignant conversion of benign tumours, but this theory has now been discarded, and most are considered to arise de-novo. ${ }^{1}$ Initially, they may mimic benign conditions such as lipomas, or neurofibromas, but, rapid growth, large size, and deep location must invoke suspicion. Sarcoma occurs in a younger age group compared to carcinoma, with fleshy appearance and areas of hemorrhage and necrosis. It shows rapid growth, with a tendency for the early hematogenous spread. Lymphatic spread is rare.

We report an interesting case of an inguinal canal leiomyosarcoma that was a clinical conundrum due to its sheer rarity. ${ }^{2}$ The clinical presentation, investigative findings, and management are discussed along with relevant works of literature.

\section{CASE PRESENTATION}

A 70-year-old male with insignificant past medicosurgical history presented with one month of insidious onset swelling in the right inguinal region (Fig. 1). It was firm to touch. The swelling was nontender; it was free from the skin and had restricted mobility over the underlying structures. The trans-illumination test was negative, and the swelling was irreducible. There was no palpable inguinal or abdominal lymphadenopathy. He had no associated fever, nausea, or loss of appetite. There were no urinary symptoms or bowel irregularities. The digital rectal examination was also normal. Hematological and biochemical tests and urinalysis were normal. On sonography of the abdomen and right inguinal

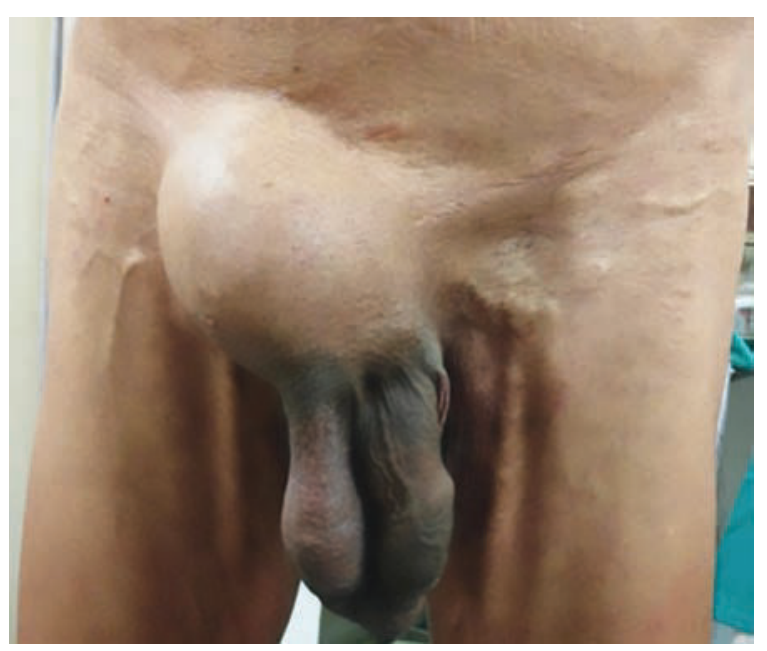

Fig. 1: A lump is visible over the right inguinal region. The swelling has led to the deviation of penis to the opposite side 


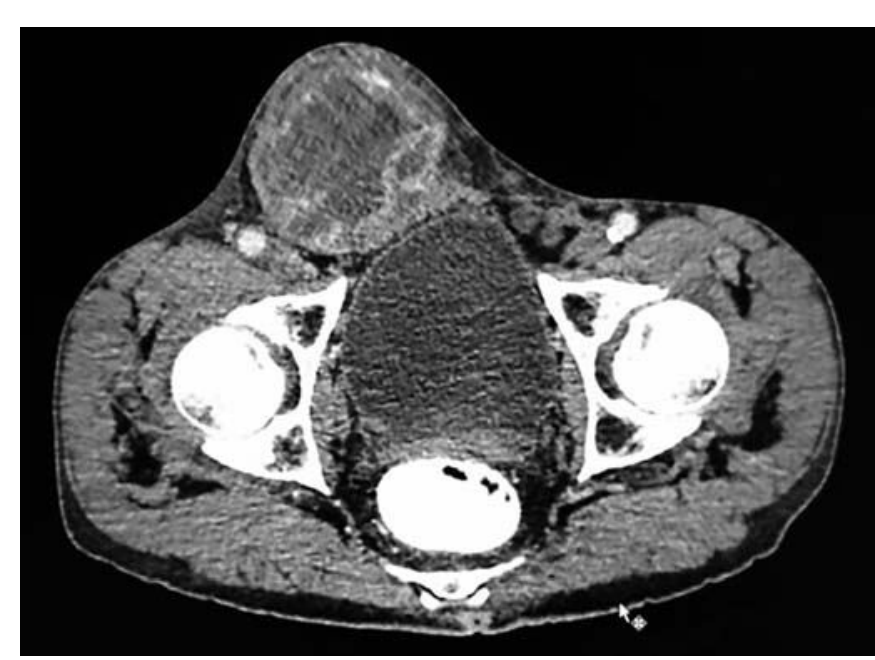

Fig. 2: CT scan of abdomen and pelvis which shows a heterogeneous mass arising from the right inguinal canal.

region, a heterogenous globular lesion $7 \times 8 \times 8 \mathrm{~cm}$ in size was seen arising from the inguinal canal. The cord structures could not be visualized clearly. Radiologist's impression was that of a lymph nodal mass, possibly a lymphoma. Abdomen and testis were normal. CT scan of the abdomen and pelvis was done and showed similar findings, with no evidence of metastasis (Fig. 2). FNAC was done, but the smear did not identify any representative tissue. A working diagnosis of a lymph nodal mass was made and the patient posted for surgery.

Using the inguinal approach an excisional biopsy was performed. A globular, light brown, well circumscribed, the fleshy tumour was seen originating from the spermatic cord sheath. The cord structures could be easily separated and preserved. The testis was delivered into the operative field and found to be grossly normal and preserved (Fig. 3). The anatomical closure was done, and the specimen sent for histopathological examination (HPE).

Microscopically, the growth from the spermatic cord was composed of smooth muscle-like cells arranged in a whorled pattern with mitotic figures, nuclear polymorphism, and hemorrhage as shown in Figure 4. Immunohistochemistry showed positive staining with smooth muscle actin (SMA) and desmin. Findings were consistent with grade II leiomyosarcoma. The patient had an uneventful recovery and was discharged on the 4th postoperative day. He was advised to review a week later at the outpatients' department, but he did not turn up. All possible means were used to trace him, but he remained inaccessible and was lost to follow-up.

\section{DISCUSSION}

It is a common clinical situation to have a patient presenting with a swelling in the groin. Most of the time such a swelling is an inguinal hernia, hydrocele of the cord, lymph nodal mass or a saphena varix. Rarely one may encounter an undescended testis. Such swellings are of an indolent nature and can be easily treated surgically.

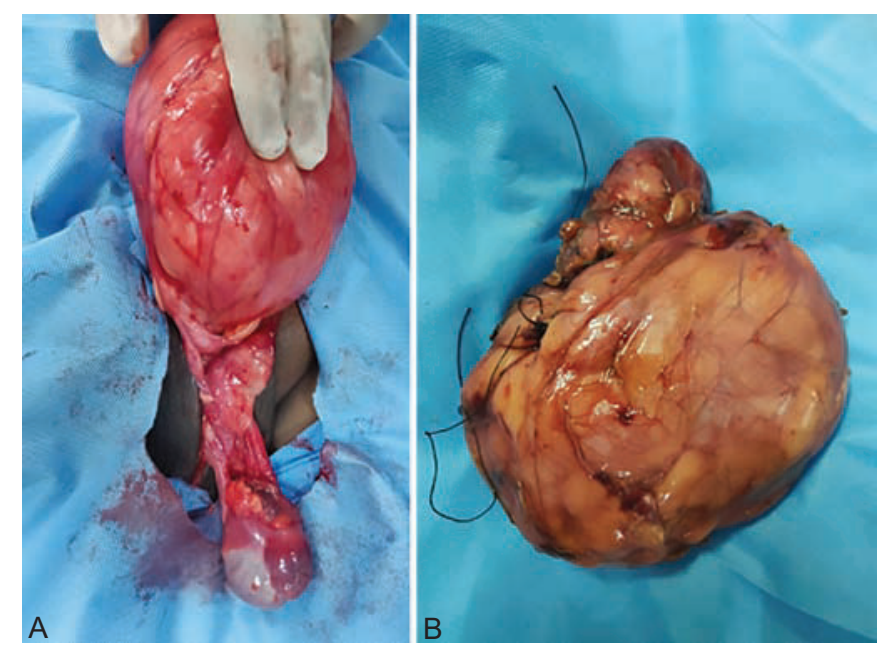

Figs 3 A and B: (A) The intraoperative image of a mass arising from the spermatic cord with a normal testis seen separately; (B) Macroscopic appearance of the tumour

According to previous studies, the prevalence of an inguinal hernia is nearly $5 \%$ worldwide. ${ }^{3}$ In the United States, 700,000 herniorrhaphy procedures are performed annually, which shows the high prevalence of the disease. ${ }^{4}$ Understandably, a swelling in the inguinal region would also point towards the diagnosis of an inguinal hernia first, rather than a soft tissue tumour.

In our case, despite our long-term experience in treating inguinal conditions, finding an inguinal canal malignancy was entirely unanticipated.

However, an inguinal swelling without reducibility or cough impulse did evoke suspicion. Also, an inconclusive FNAC further added to the confusion. Ultrasonogram (USG) and CT scan helped in the localization of the origin and consistency of the swelling. Intraoperatively a fleshy growth from the spermatic cord sheath brought the diagnosis closer to a soft tissue sarcoma which was after that confirmed on HPE.

Soft tissue sarcomas are rare malignancies and account for $<1 \%$ of the overall human burden of malignant tumours, and yet they remain life-threatening, and

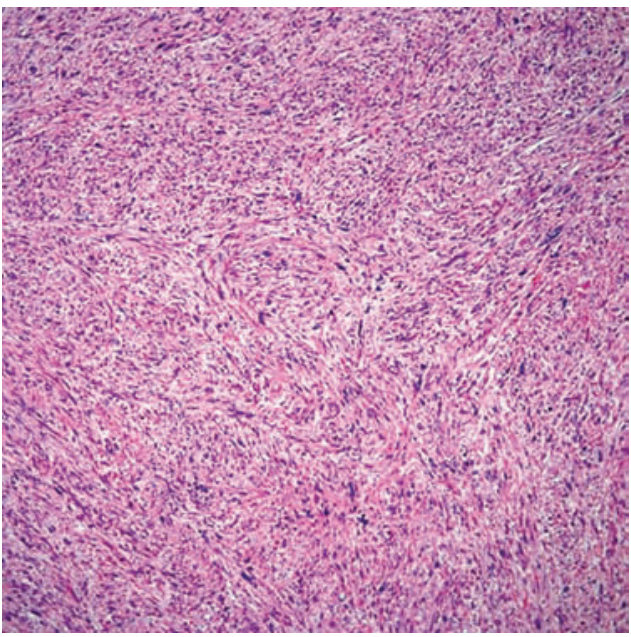

Fig. 4: Microscopic appearance of leiomyosarcoma (stained with eosin/haematoxylin stain) 
approximately $40 \%$ of patients with newly diagnosed soft tissue sarcoma die of the disease. ${ }^{5}$

Early soft tissue sarcoma, is highly curable, but if diagnosed at the time of extensive local or metastatic disease, it is rarely curable. The relatively small number of cases and the great diversity in histopathologic features have made a comprehensive understanding of these disease entities difficult. ${ }^{1}$

Benign mesenchymal tumours are 100-fold more common than soft tissue sarcomas. ${ }^{6}$ The annual international incidence of soft tissue sarcoma is estimated to be between 1.5 and 5.0 cases per 100,000. Usually, incidence increases progressively with age from approximately 1 to 2 per 100,000 at age 15, to approximately 6 per 100,000 at age 49 , and to as high as approximately 20 per 100,000 at age $80.7,8$

Most soft tissue sarcomas are believed to be sporadic and have no clearly defined cause. In a small proportion of cases, researchers have identified predisposing or associated factors, including genetic factors, lymphedema, prior radiation therapy, and carcinogens.

Genetic factors like germline mutations in the APC gene, or the NF1 gene are responsible. Li-Fraumeni syndrome is a rare, highly penetrant familial cancer phenotype usually associated with germline mutations in $T P 53^{9}$ and the index tumours in $36 \%$ of such patients are soft tissue or bone sarcomas of diverse histology. Lymphedema is best-recognized in association with the postmastectomy, post-irradiated lymphedematous arm. ${ }^{10}$ Radiation-associated sarcomas are most often seen in diseases commonly treated with radiotherapy and in those in which patient survival is typically long for example breast cancer, lymphoma, genitourinary cancer, and head and neck malignancies. ${ }^{11}$ Trauma as a predisposing factor is controversial. Desmoid tumours of the abdomen commonly follow childbirth and may be located in the bed of prior surgery. ${ }^{12}$ Chemical carcinogens have an established role in the development of hepatic angiosarcomas, such as vinyl chloride, thorotrast, and arsenic, to name a few. ${ }^{13}$

Soft tissue sarcomas can occur in any site throughout the body. A total of $45 \%$ are located in the extremities, with $30 \%$ of all lesions occurring in the lower limb (most commonly in the thigh); $38 \%$ are intra-abdominal; $10 \%$ are truncal, and $5 \%$ are head and neck. ${ }^{1}$ Leiomyosarcoma account for just 5 to $10 \%{ }^{2}$ of which, leiomyosarcoma originating from the spermatic cord are the commonest followed by those in the scrotum and the epididymis. ${ }^{14}$

Paratesticular leiomyosarcoma is an extremely rare tumour with approximately 110 cases reported in literature worldwide. ${ }^{15}$ A PubMed search using the keywords "paratesticular", "inguinal", "leiomyosarcoma" and "India" brought out 24 research articles.

Preoperative diagnosis of inguinal leiomyosarcoma is difficult. Unsurprisingly, clinicians do not usually consider inguinoscrotal lumps to be underlying sarcomas owing to the relative lack of prevalence compared with a hernia. Our case highlights the importance of being aware of such lesions when patients present with lumps in this region.

Clinically, they present as a painless, firm paratesticular mass. ${ }^{14}$ Preoperative ultrasonography of an inguinal lump is useful and should be extended to the scrotal region to reveal the relationship to the testis. However, even if a discrete lump is identified preoperatively, diagnosis is usually not made until histological analysis. An incisional or excisional biopsy has the highest scope for accurate histological diagnosis. ${ }^{1}$ Rarely it may present as a cause for limb lymphedema ${ }^{16}$ or metastasis to the scalp. ${ }^{17}$

The most common mode is lymphatic spread, followed by hematogenous and then local invasion. ${ }^{14} \mathrm{Lymph}$ nodes and lung fields (for hematogenous spread) are investigated with CT, and local invasion can be explored with magnetic resonance imaging (MRI). In our case MRI was not done as the information from the CT scan was deemed enough.

Radical orchidectomy with high ligation of the spermatic cord is believed widely to be the treatment of choice. ${ }^{18}$ Recent trends favor the preservation of vital structures, if not continuously involved with a tumour. Hence a testis preserving surgery was considered suitable in our case. As per the latest American Joint Committee on Cancer (AJCC) guidelines for soft tissue sarcomas ${ }^{19}$ the malignancy in question falls under Stage IIIA. The proposed treatment as per the National Comprehensive Cancer Network (NCCN) 2018 guidelines $^{20}$ is a tumor excision with oncologically acceptable margins with or without adjuvant radiotherapy.

Often, these case are mismanaged because of the inadequate experience of dealing with inguinal malignancies, as was the case with nine patients reported by Collin et al. ${ }^{21}$

\section{CONCLUSION}

It is recognized that the diagnosis of groin sarcoma is complicated by the fact that sarcoma is probably the least likely explanation for a lump in the groin or inguinal region. Five patients in a series by Fieber et al. were initially diagnosed with a 'hernia' and underwent herniorrhaphy before the correct diagnosis was realized. ${ }^{21}$ Sarcomas may be misdiagnosed, ${ }^{22,23}$ in this way, or may co-exist with hernias ${ }^{24}$ in the form of a cord liposarcoma. Less than $0.1 \%$ of hernia operations are considered to be complicated by this phenomenon. ${ }^{25}$ Other reports suggest that unexpected tumours are found in only $0.00098 \%$ of specimens from hernia operations. ${ }^{26}$

Preoperative clinical findings of fixed or rubbery masses in the inguinal canal may prompt further investigation, as this seems to be a common attribute in the cases inadvertently explored in certain series. ${ }^{21}$

It would be wise for the young doctors to remember that all swellings in the inguinal region may not be hernias. 


\section{ABBREVIATIONS}

USG : Ultrasonogram

CT : Computed tomography

MRI : Magnetic resonance imaging

FNAC : Fine needle aspiration cytology

AJCC : American Joint Committee on Cancer

NCCN : National Comprehensive Cancer Network

\section{CONSENT}

Written informed consent was obtained from the patient for publication of this case report and accompanying images.

\section{AUTHORS' CONTRIBUTIONS}

Rahul Goel participated in the surgical and perioperative management of the patient and conception and design of the report and wrote the paper. Kasiviswanathan M performed the USG and CT scan of the patient.

\section{ACKNOWLEDGMENTS}

Authors would like to thank the ward staff of the hospital and the Department of Pathology for providing support and helping in the management of the patient.

\section{REFERENCES}

1. DeVita Jr V T, Lawrence TS., Rosenberg SA. Soft Tissue Sarcoma. In: Cancer: Principles \& Practice of Oncology. 10th ed. Wolters Kluwer; 2015. pp 1253-1291.

2. Goldblum JR, Weiss SW, Folpe AL. Enzinger and Weiss's Soft Tissue Tumours, 6th Edition. Enzinger and Weiss"s Soft Tissue Tumours. 2013.

3. Dattola P, Alberti A, Dattola A, Giannetto G, Basile G, Basile M. Inguino-crural hernias: preoperative diagnosis and postoperative follow-up by high-resolution ultrasonography. A personal experience. Ann Ital Chir. 2002;73(1):65-68.

4. Rutkow IM. Open versus laparoscopic groin herniorrhaphy: economic realities. Inguinal Hernia Adv or Contraversies. 1994;

5. Jemal A, Siegel R, Ward E, Hao Y, Xu J, Thun M. Cancer statistics, 2009. CA Cancer J Clin [Internet]. 2009;59(4):225-249.

6. Rydholm A, Berg NO. Size, site and clinical incidence of lipoma: Factors in the differential diagnosis of lipoma and sarcoma. Acta Orthop. 1983;54(6):929-934.

7. Toro JR, Travis LB, Hongyu JW, Zhu K, Fletcher CDM, Devesa SS. Incidence patterns of soft tissue sarcomas, regardless of primary site, in the Surveillance, Epidemiology and End Results program, 1978-2001: An analysis of 26,758 cases. Int J Cancer. 2006;119(12):2922-2930.

8. Wu X, Groves FD, McLaughlin CC, Jemal A, Martin J, Chen VW. Cancer incidence patterns among adolescents and young adults in the United States. Cancer Causes Control [Internet]. 2005;16(3):309-320.

9. Malkin D, Li FP, Strong LC, Fraumeni JF, Nelson CE, Kim $\mathrm{DH}$, et al. Germ line p53 mutations in a familial syndrome of breast cancer, sarcomas, and other neoplasms. Science. 1990 Nov 30;250(4985):1233-1238.

10. Stewart FW, Treves N. Lymphangiosarcoma in postmastectomy lymphedema. A report of six cases in elephantiasis chirurgica. Cancer. 1948;1(1):64-81.

11. Gladdy RA, Qin LX, Moraco N, Edgar MA, Antonescu $\mathrm{CR}$, Alektiar KM, et al. Do radiation-associated soft tissue sarcomas have the same prognosis as sporadic soft tissue sarcomas? J Clin Oncol. 2010;28(12):2064-2069.

12. Fong $Y$, Rosen PP, Brennan MF. Multifocal desmoids. Surgery. 1993;114(5):902-906.

13. Zahm SH, Fraumeni Jr. JF. The epidemiology of soft tissue sarcoma. Semin Oncol [Internet]. 1997;24(5):504-514.

14. Dangle P, Basavaraj DR, Bhattarai S, Paul AB, Biyani CS. Leiomyosarcoma of the spermatic cord: case report and literature review. Can Urol Assoc J [Internet]. 2007;1(1):55-58.

15. Vogelaar FJ, Schuttevaer HM, Willems JM. A patient with an inguinal mass: A groin hernia? Neth J Med. 2009;67(11):399400.

16. Abd Kadir HK, Naik J, Chandrasekar CR. Leiomyosarcoma presenting as "idiopathic" unilateral lower limb lymphoedema. BMJ Case Rep. 2017 Oct;2017.

17. Soipi S, Vucic M, Ulamec M, Tomas D, Kruslin B, Spajic B. Leiomyosarcoma of the spermatic cord with scalp metastasis: case report and literature review. Coll Antropol. 2014 Jun;38(2):763-766.

18. Rodríguez D, Olumi AF. Management of spermatic cord tumours: a rare urologic malignancy. Ther Adv Urol [Internet]. 2012;4(6):325-334.

19. Bochner BH, Hansel DE, Efstathiou JA, Konety B, Lee C.T, Mckiernan JM. AJCC Cancer Staging Manual [Internet]. AJCC Cancer Staging Manual. 2017. 758-764 p.

20. Mehren MV, Randall RL, Benjamin RS, Boles S, Bui MM, Ganjoo KN, et al. Soft tissue sarcoma, version 2.2018: Clinical practice guidelines in oncology. JNCCN J Natl Compr Cancer Netw. 2018;16(5):536-563.

21. Collin T, Blackburn A V, Milner RH, Gerrand C, Ragbir M. Sarcomas in the groin and inguinal canal - Often missed and difficult to manage. Ann R Coll Surg Engl. 2010;92(4):326-329.

22. Bell RS, O'Sullivan B, Mahoney JL, Nguygen C, Langer F, Catton C. The inguinal sarcoma: A review of five cases. Can J Surg. 1990;33(4):309-311.

23. Geelhoed GW, Millar RC, Ketcham AS. Hernia presentation of cancer in the groin. Surgery [Internet]. 1974;75(3):436-441.

24. Fieber SS, Wolstenholme JT. Primary tumours in inguinal hernial sacs. AMA Arch Surg. 1955 Aug;71(2):254-256.

25. Montgomery E, Buras R. Incidental liposarcomas identified during hernia repair operations. J Surg Oncol. 1999;71(1):5053.

26. Kassan MA, Munoz E, Laughlin A, Margolis IB, Wise L. Value of routine pathology in herniorrhaphy performed upon adults. Surg Gynecol Obs [Internet]. 1986;163(6):518-522. 